\title{
Finding a Pair on a Mesh with Multiple Broadcasting is Hard
}

\author{
Peter Damaschke \\ FernUniversität, Theoretische Informatik II \\ 58084 Hagen, Germany \\ Peter.Damaschke@fernuni-hagen.de
}

\begin{abstract}
We show that finding two items at unknown positions in a. square mesh with exclusive-write row and column buses is as hard as semigroup computation on this architecture, i.e. it requires $\Omega\left(n^{1 / 6}\right)$ time in the worst case. Consequently, the time complexity of any sensitive computational problem with sparse input scattered on the mesh depends on the number of processors rather than on the input size. A slightly modified proof yields the tight lower bound for computing the logical OR on the mesh, even if the input is known to contain at most two 1's.
\end{abstract}

\section{Introduction}

The $\sqrt{n} \times \sqrt{n}$ mesh with row and column buses is a well-studied architecture for parallel computing, both as a theoretical model and as a practical architecture. It is particularly suitable for parallel image processing where each processor represents a pixel or a group of pixels forming a small square of the picture. Starting with [12], optimal mesh algorithms have been developed for a lot of fundamental computational problems. In the references we listed a selection of papers where one can find many of these results and further hints.

The usual assumption is that the input is distributed on the whole mesh, one data item per processor. That means, the input size is equal to the number $n$ of processors, and the complexities of algorithms are functions of $n$. Some problems have also been studied for sparse inputs, i.e. for $k$ data items where $k$ is small compared to $n$, and the complexities are estimated as functions of $k$ rather than $n$. In these cases, it has been assumed that the input initially stands in a distinguished region of the mesh, e.g. in the first $k / \sqrt{n}$ rows (as in [2]), or locally sparse inputs are considered [13].

In contrast to this, in the present paper we consider the situation that a sparse input is scattered on the mesh, but we do not have any knowledge about the positions of the $k$ data items beforehand.

This type of problems may really occur in image processing: For instance, suppose that we wish to perform any image processing operation on pictures of a video such that the number of significant pixels in each picture is typically small;' but nevertheless the significant pixels cover the entire mesh during the video. (E.g. think of line drawings or sporadic small objects which are moving.) Then it would be desirable to have mesh algorithms whose complexities depend 
only on the input size $k$ rather than on the mesh size $n$, although the $k$ input positions are arbitrary and previously unknown.

Whether such algorithms can exist or not does strongly depend on the write conflict resolution rule on the buses. (Similar effects are also known in the PRAM model [7].) If concurrent write access to the buses is strictly prohibited then the problem of semigroup computation with $k=2(!)$ non-zero items in unknown positions on the mesh is already as hard as semigroup computation with $n$ arbitrary summands, namely, it requires $\Omega\left(n^{1 / 6}\right)$ time. Semigroup computation means: Each processor contains an element from a fixed commutative semigroup, and the goal is to compute their sum and to send it to some distinguished target processor.

The contribution of this paper is a proof of this lower bound. We have already announced this result in [6], but we did not publish the proof anywhere. In [6] we demonstrated that problems with sparse inputs on the mesh are solvable in a time bound depending only on $k$ (independent of $n$ ), provided that several processors may write simultaneously the same item onto the same bus. (This is the Common CRCW rule). Particularly we proved a $O\left(k^{1 / 4} \log ^{2} k\right)$ bound for semigroup computation with $k$ non-zero items.

In Section 2 we recall some basics about enhanced meshes, and in Section 3 we give the lower bound proof. Before this we would like to emphasize some aspects of the result:

(1) The $\Omega\left(n^{1 / 6}\right)$ lower bond for full semigroup computation (with $n$ items) in [12] uses a quite simple counting argument, based on fact that the sum can depend on each of the $n$ summands. However, our present proof shows that the communication restrictions are the more essential reason for the lower bound, rather than the number of meaningful summands: Only two non-zero summands require the same time complexity. We may even assume that the processors have unbounded local memory and computational power.

(2) For our presentation we have chosen the semigroup problem because of its very simple formulation, but one can easily note that the idea of the lower bound proof applies to any sensitive computational problem on the mesh. (Here sensitive means that the output can depend on every input item.) A slight modification of our proof immediately gives an $\Omega\left(n^{1 / 6}\right)$ bound for the logical $\mathrm{OR}$, even if we previously know that at most two input items have value 1 and all others are 0.

\section{The Computational Model}

For brevity we can only give an informal description, but it should not be hard to specify the model formally.

The mesh is an array of $n$ processors, arranged as a $\sqrt{n} \times \sqrt{n}$ grid. Each processor knows its own position in the mesh. Each processor is connected to its four neighbors (of course, except the processors at the borders and corners which have three or two neighbors) by local links. Additionally, each row and column is enhanced by a bus. 
The processors work in synchronized steps. Each processor knows the algorithm to be executed. In any step, each processor can perform local computations with its contents, send messages to its neighbors and receive messages through the local links. It can also write a message onto its row or column bus and read the messages from these buses. A bus can broadcast messages originated by only one processor each time, but all processors along the bus can simultaneously read. More precisely, we assume that concurrent write access to a bus is strictly prohibited; whenever this happens, the computation is aborted unsuccessfully (CREW rule).

All these computations and communications are done according to the algorithm to be executed. In particular, a correct algorithm for some problem must exclude write conflicts on buses, for any possible input.

Note that the actions of a processor in any step may depend on the entire processor history up to this moment, that is: on the own coordinates, the own initial contents, and the sequence of all received messages. We assume unbounded local memory, as well as unbounded computational power of each processor in any step. Since we are going to prove a lower bound we can make such concessions.

By the distance $d(p, q)$ of two processors $p, q$ we always mean their Manhattan distance in the mesh. For a subset $G$ of processors, $d(p, G)$ denotes the minimum distance $d(p, r)$ for $r \in G$.

\section{Finding a Pair is Hard}

Theorem 1. Consider the following problem: Two non-zero elements of a semigroup stand in unknown processors of the $\sqrt{n} \times \sqrt{n}$ mesh with CREW buses, whereas all other processors contain zero. Then any algorithm computing the sum of these items requires $\Omega\left(n^{1 / 6}\right)$ time.

In the remainder of the paper we shall prove this theorem. So consider a fixed algorithm for computing the sum of two semigroup elements being initially located in two unknown processors $p$ and $q$. (We briefly speak of an input in $p$ "and q.) We will show that the algorithm needs $\Omega\left(n^{1 / 6}\right)$ steps for some particular input, or even works incorrectly.

For any step $t=0,1,2,3, \ldots$ let us inductively define sets of processors called $g^{-}(t), g^{l}(t)$, and $G(t)$, as follows: Let be $g^{-}(0)=g^{l}(0)=G(0)=\emptyset$, and denote by $g^{-}(t+1)$ the set of all processors $r$ satisfying the following conditions: There exists an input in some pair of processors $p, q$ with $d(p, q)>2 t, d(p, G(t))>t$, $d(q, G(t))>t$, such that processor $r$ writes some message onto its row bus in step $t+1$. The set $g \mathrm{l}(t+1)$ is defined analogously with "column bus" instead of "row bus". Finally let be

$$
G(t)=\bigcup_{i=0}^{t}\left(g^{-}(t) \cup g^{l}(t)\right) .
$$

Note that the above sets are well-defined. An input in $p, q$ is said to be hidden in step $t$ if it satisfies the above three distance inequalities $d(p, q)>2 t$, $d(p, G(t))>t, d(q, G(t))>t$. 
The next lemma is an obvious but useful reformulation of these definitions.

Lemma 2. Consider an input in $p, q$ that is still hidden in step $t$, and run the given algorithm on this input. Then any processor $r$ that has written some message onto its row or column bus up to step $t$ belongs to $G(t)$.

Proof. Suppose that processor $r$ sent some message to its row or column bus in step $i \leq t$. Clearly, the considered input was hidden in step $i$. Hence $r$ satisfies the definition of a member of $g^{-}(i)$ or $g^{\prime}(i)$ which implies $r \in G(t)$.

Hidden inputs immediately provide lower bounds:

Lemma 3. For any hidden input in step t, the given algorithm cannot compute the sum of the two items before step $t$.

Proof. Through local links only, informations about the summands in $p$ and $q$ can have been sent only to processors with distance at most $t$ to $p$ and $q$, respectively. None of these processors belongs to $G(t)$. On the other hand, Lemma 2 says that all processors that have broadcast any informations through buses so far belong to $G(t)$. We conclude that no information about the summands has been broadcast by buses. Moreover, no processor has distance at most $t$ to both $p$ and $q$. Altogether, no processor has received both summands up to step $t$.

To become more concrete, we next consider a class of algorithms fulfilling a certain condition that enables us to prove the asserted lower time bound. This condition itself might appear somewhat artificial, but afterwards we will see that any algorithm violating this condition is incorrect. Altogether this will prove Theorem 1 . In the following we omit \lfloor\rfloor brackets for convenience.

Lemma 4. Suppose that, for each $t$ and each row $R$ of the mesh, $g^{-}(t+1) \cap R$ is completely contained in some consecutive segement of $R$ with length at most $4 t$, and analogously for the columns and the $g^{l}(t+1)$. Then there exist inputs still being hidden in step $t=0.2 n^{1 / 6}$.

Proof. Since we have $2 n^{1 / 2}$ rows and columns and $t=0.2 n^{1 / 6}, G(t)$ is contained in at most $0.4 n^{2 / 3}$ segments each of length at most $4 t \leq 0.8 n^{1 / 6}$. Partition the mesh into $n^{2 / 3}$ submeshes of size $n^{1 / 6} \times n^{1 / 6}$. Clearly, each of the segments can meet at most two of these submeshes, hence at least $0.2 n^{2 / 3}$ submeshes remain disjoint to $G(t)$. Now it is obvious that we can fix two processors $p$ and $q$ in these free regions, satisfying the above three distance inequalities. Hence an input located in $p, q$ is hidden in step $t$.

In view of Lemma 3 this yields the $\Omega\left(n^{1 / 6}\right)$ lower bound. It remains to consider algorithms violating the premises of Lemma 4 . Without loss of generality we assume that, for some $t$, there exist two processors $r, s \in g^{-}(t+1)$ in the same row of the mesh, such that $d(p, q)>4 t$. Moreover we may consider the first such $t \leq 0.2 n^{1 / 6}$. Under this condition we prove: 
Lemma 5. There exists a hidden input in some pair of processors $p, q$ such that both $r$ and $s$ write onto the row bus in step $t+1$, thus violating the CREW rule.

Proof. We will argue somewhat informally to keep the presentation short. Since $r \in g^{-}(t+1)$ and $s \in g^{-}(t+1)$, there exist two (in general different!) hidden inputs in processor pairs $p_{r}, q_{r}$ and $p_{s}, q_{s}$, respectively, as in the definition of $g^{-}(t+1)$. The former input would force $r$ to write onto the row bus in step $t+1$, the latter input would do the same with $s$. Now we claim that there also exists one hidden input that forces both $r$ and $s$ to write simultaneously onto the bus.

For constructing such a malicious input, we first introduce some notion: Processor $r$ is called internal if $d\left(r, p_{r}\right) \leq t$ or $d\left(r, q_{r}\right) \leq t$, otherwise $r$ is called external. Analogously, processor $s$ is said to be internal or external with respect to $p_{s}, q_{s}$. Now three cases are possible; the fourth case is symmetric.

Let both $r$ and $s$ be internal, without loss of generality assume $d\left(r, p_{r}\right) \leq t$ and $d\left(s, p_{s}\right) \leq t$. Since $d(r, s)>4 t$, the "balls" (with respect to the Manhattan distance) with centers $p_{r}$ and $p_{s}$ and radius $t$ are disjoint. Since we consider hidden inputs, we can easily conclude from Lemma 2 that the history of $r$ until step $t$ only depends on the input item in $p_{r}$, and similarly, the history of $s$ only depends on the input item in $p_{s}$. Hence the pair $p_{r}, p_{s}$ together with the original input items has the asserted bad property.

Let be $r$ internal and $s$ external. We similarly conclude that the history of $s$ until step $t$ is independent of the summands of the input in $p_{s}, q_{s}$ since $s$ cannot have received any information about them. Hence we can replace $p_{s}, q_{s}$ by an arbitrary other hidden input for which $s$ is external, without changing the behaviour of $s$ in the first $t$ steps. Particularly, we can choose $p_{r}$ with the original semigroup element in it, and an arbitrary new $q$ with distance more than $t$ from $s$. (By the estimation from Lemma 4 such positions exist.) As in the previous case, this alteration does not affect the behaviour of $r$, too. Hence both $r$ and $s$ will now write simultaneously onto the row bus.

Finally, if $r$ is external with respect to $p_{r}, q_{r}$ and $s$ is external with respect to $p_{s}, q_{s}$ then choose an arbitrary new hidden input for which both $r$ and $s$ are external.

\section{References}

1. A.Bar-Noy, D.Peleg: Square meshes are not always optimal, IEEE Trans. on Computers 40 (1991), 196-204

2. D.Bhagavathi, S.Olariu, W.Shen, L.Wilson: A unifying look at semigroup computations on meshes with multiple broadcasting, Parallel Architectures and Languages Europe PARLE'93, Munich 1993, LNCS 694, Springer, 561-569

3. V.Bokka, H.Gurla, S.Olariu, J.L.Schwing, I.Stojmenovic: Time-optimal digital geometry algorithms on meshes with multiple broadcasting, 3rd Int. Workshop on Par. Image Analysis, College Park/ MD 1994, 199-213

4. Y.C.Chen, W.T.Chen: Efficient median finding and its application to two-variable linear programming on mesh-connected computers with multiple broadcasting, Journal of Par. and Distr. Computing 15 (1992), 97-84 
5. Y.C.Chen, W.T.Chen, G.H.Chen, J.P.Sheu: Designing efficient parallel algorithms on mesh-connected computers with multiple broadcasting, IEEE Trans. PADS 1 (1990), 241-246

6. P.Damaschke: Coping with sparse inputs on enhanced meshes - semigroup computations with common CRCW buses, 10th Int. Parallel Proc. Symposium IPPS'96, Honolulu 1996, IEEE Press, 682-686

7. F.E.Fich, P.Ragde, A.Wigderson: Simulations among concurrent-write PRAMs, Algorithmica 3 (1988), 43-51

8. H.Gurla: Leftmost one computation on meshes with row broadcasting, Info. Proc. Letters 47 (1993), 261-266

9. K.Iwama, E.Miyano, Y.Kambayashi: Routing problems on the mesh of buses, 3rd Int. Symposium on Algorithms and Computation ISAAC'92, Nagoya 1992, LNCS 650, Springer, 155-164

10. J.Y.T.Leung, S.M.Shende: On multidimensional packet routing for meshes with buses, Journal of Par. and Distr. Computing 20 (1994), 187-197

11. S.Olariu, I.Stojmenovic: Time-optimal nearest-neighbor computations on enhanced meshes, Parallel Architectures and Languages Europe PARLE'94, Athens 1994, LNCS 817, Springer, 134-145

12. V.K.Prasanna Kumar, C.S.Raghavendra: Array processor with multiple broadcasting, Journal of Par. and Distr. Computing 4 (1987), 173-190

13. V.K.Prasanna Kumar, D.I.Reisis: Image computations on meshes with multiple broadcast, IEEE Trans. PAMI 11 (1989), 1194-1201

14. S.Rajasekaran: Mesh connected computers with fixed and reconfigurable buses: packet routing, sorting, and selection, 1st European Symposium on Algorithms ESA'93, Bad Honnef 1993, LNCS 726, Springer, 309-320

15. J.F.Sibeyn, M.Kaufmann, R.Raman: Randomized routing on meshes with buses, 1st European Symposium on Algorithms ESA'93, Bad Honnef 1993, LNCS 726, Springer, 333-344 